\title{
Cystoscopic findings in women with minimal and maximal lower urinary tract symptoms
}

\author{
Ioana Marcu ${ }^{1}$, Jeffrey A. Gavard ${ }^{1}$, Golnar Vazirabadi ${ }^{1}$, Joe Y. Shi ${ }^{1}$, Andrew C. Steele ${ }^{2}$, Fah Che Leong ${ }^{2}$, \\ Mary T. McLennan ${ }^{2}$, Jennifer A. Bickhaus ${ }^{2}$, Mary B. Holloran-Schwartz ${ }^{3}$, Patrick Yeung Jr ${ }^{3}$, Eugen C. Campian ${ }^{2}$ \\ ${ }^{1}$ St. Louis University School of Medicine, Department of Obstetrics, Gynecology, and Women's Health, St. Louis, MO, USA; ${ }^{2}$ St. Louis University \\ School of Medicine, Division of Female Pelvic Medicine and Reconstructive Surgery, Department of Obstetrics, Gynecology, and Women's Health, \\ St. Louis, MO, USA; ${ }^{3}$ St. Louis University School of Medicine, Division of Minimally Invasive Gynecologic Surgery, Department of Obstetrics, \\ Gynecology, and Women's Health, St. Louis, MO, USA \\ Contributions: (I) Conception and design: All authors; (II) Administrative support: I Marcu, JA Gavard, G Vazirabadi, MT McLennan, JA Bickhaus, \\ EC Campian; (III) Provision of study materials or patients: I Marcu, JA Gavard, G Vazirabadi, AC Steele, FC Leong, MT McLennan, MB Holloran- \\ Schwartz, P Yeung Jr, EC Campian; (IV) Collection and assembly of data: I Marcu, JA Gavard, G Vazirabadi, JY Shi, AC Steele, FC Leong, MT \\ McLennan, MB Holloran-Schwartz, P Yeung Jr, EC Campian; (V) Data analysis and interpretation: I Marcu, JA Gavard, JA Bickhaus, EC Campian; \\ (VI) Manuscript writing: All authors; (VII) Final approval of manuscript: All authors. \\ Correspondence to: Ioana Marcu, MD. Saint Louis University School of Medicine, Department of Obstetrics, Gynecology, and Women's Health, \\ Division of Female Pelvic Medicine and Reconstructive Surgery, 6420 Clayton Rd. St. Louis, MO 63117, USA. Email: imarcu2000@gmail.com.
}

Background: Glomerulations are not specific for interstitial cystitis/bladder pain syndrome (IC/BPS). Controversy exists about whether cystoscopic findings differ between patients with and without lower urinary tract symptoms. We sought to compare the prevalence of cystoscopic findings in women with "no or minimal" urinary symptoms to those with a "high" symptom burden.

Methods: This is a secondary analysis of a prospective cohort study performed at a University Educational Facility. Participants in this study were part of a larger prospective study, in which female patients scheduled to undergo routine gynecologic procedures were all consented for cystoscopy with hydrodistension (CWHD). We defined the "minimally symptomatic group" as those with $\leq 1$ on each of the O'Leary/Sant Interstitial Cystitis Symptom Index (ICSI) subscores and without history of IC/BPS. The "highly symptomatic" cohort of women had composite ICSI score $\geq 12$ and a Burning/Pain subscore of 4 or 5 . All were non-smokers.

Results: A total of 84 women underwent CWHD, with 33 having minimal symptoms and 51 being highly symptomatic. The two groups were not statistically different when assessing for 'any glomerulations' compared to 'no glomerulations.' However, minimally symptomatic women had an eight-fold lower prevalence of significant glomerulations than highly symptomatic women $(3.0 \%$ minimally symptomatic $v s$. $23.5 \%$ highly symptomatic, $\mathrm{P}<0.05$.)

Conclusions: Extensive glomerulations ( $\geq 10$ in 3 or 4 quadrants) are rare in women with minimal urinary symptoms. These findings contrast with prior limited prospective data which quoted similar incidence of glomerulations in IC/BPS patients and asymptomatic patients. This study highlights the importance of evaluating objective evidence on CWHD and merits further investigation as part of the ongoing conversation regarding the definition of bladder health and pathology

Keywords: Asymptomatic; cystoscopy with hydrodistension; glomerulations; interstitial cystitis; pelvic pain

Submitted Mar 07, 2021. Accepted for publication May 25, 2021.

doi: 10.21037/tau-21-195

View this article at: https://dx.doi.org/10.21037/tau-21-195

$\wedge$ ORCID: 0000-0002-4689-3065. 


\section{Introduction}

Glomerulations on cystoscopy with hydrodistension (CWHD) have been classically associated with Interstitial Cystitis/Bladder Pain Syndrome (IC/BPS), a chronic irritative bladder syndrome $(1,2)$. However, there is increasing evidence that glomerulations are non-specific for IC/BPS (3-6).

The presence of significant glomerulations were once considered necessary to the diagnosis of IC/BPS. The National Institute of Diabetes and Digestive and Kidney Diseases (NIDDK) definition of IC/BPS was used pervasively used in early research and requires the presence of either Hunner ulcers or of at least 10 glomerulations in 3 or 4 quadrants on cystoscopic examination, and pain associated with either filling of the urinary bladder or urinary urgency (7). In 1999, Hanno et al. found that although $90 \%$ of patients meeting the NIDDK definition for IC/BPS were also diagnosed with IC/BPS based on experienced physicians' clinical judgment, the research definition excluded more than $60 \%$ of patients judged by clinicians as having IC/BPS (8). This prompted debate regarding the clinical applicability of the NIDDK definition. Multiple studies have found that glomerulations can be found in patients with alternative diagnoses to IC/BPS; they have been found in patients with idiopathic reduced bladder storage, other lower urinary tract symptoms (LUTS), benign prostatic hypertrophy, overactive bladder (OAB), prostatitis, and urolithiasis (3-6,9-12).

From this emerged a paradigm shift in the diagnosis of IC/BPS. The American Urological Association (AUA) has stepped away from the requirement of cystoscopy with hydrodistention for the diagnosis of IC/BPS, which is now defined "an unpleasant sensation perceived to be related to the urinary bladder, associated with lower urinary tract symptoms of more than 6 weeks duration, in the absence of infection or other identifiable causes" (13). The European Association for Urology recommends that BPS be the umbrella term used for IC/BPS and IC be used only when pathologic features are found on cystoscopy or bladder biopsy (14). The International Continence Society (ICS) has subcategorized painful bladder symptoms: the diagnosis of IC encompasses only those with histologic findings or features of IC on cystoscopy and Painful Bladder Syndrome (PBS) instead refers to pelvic pain related to bladder filling "accompanied by other symptoms such as increased daytime and night-time frequency, in the absence of proven urinary infection or other obvious pathology" (15).
Importantly, the shift in the diagnostic criteria for IC/ BPS reflects a change in our understanding of IC/BPS from that of a painful urothelial disorder to a chronic pain disorder, sometimes with mucosal findings.

Glomerulations are no longer specific for IC/BPS, but it is unknown if they represent bladder pathology. In the setting of limited prospective data assessing glomerulations on cystoscopy with hydrodistension in asymptomatic women, our hypothesis was that minimally symptomatic women will be less likely to have any glomerulations on cystoscopy than women with a high irritative bladder symptom burden. To that aim, we examined cystoscopic findings in a prospective cohort of patients with a wide range of urinary symptoms. Included in this study are patients with minimal or no urinary symptoms and those with a high burden of lower urinary tract symptoms (LUTS). We present the following article in accordance with the STROBE reporting checklist (available at https://dx.doi. org/10.21037/tau-21-195).

\section{Methods}

We conducted a secondary analysis of a prospectivelycollected data set; participants in this study are part of a larger prospective cohort study performed between July 2015 and June 2017 in a university setting. As part of recruitment for subjects for the larger data set, all patients scheduled to undergo elective benign gynecological or urogynecologocial procedures with included either cystoscopy or CWHD, such as but not limited to excision of endometriosis, ovarian cystectomy, hysterectomy for benign indications, and laparoscopic myomectomy, were approached in the pre-operative area to participate. Participants were consented for CWHD as opposed to diagnostic cystoscopy at the time of their procedure. There was no follow up as part of this study.

Inclusion criteria for the large prospective study were as follows: females at least 18 years in age who presented for benign gynecologic procedures. All women eligible for inclusion were initially scheduled for procedures that included either cystoscopy or CWHD, under anesthesia. Patients had to be able to provide informed consent. Exclusion criteria for the study were as follows: pregnancy at time of study, planned cystotomy, incidental cystotomy at time of surgery, age $>90$, history of pelvic radiation, and previous or newly diagnosed bladder tumor. While tobacco users were included in the large cohort, we excluded 
smokers from this study, because of its reported association with IC/BPS and with cystoscopic findings (16-18).

After obtaining consent, all participants in the prospective study completed a series of questionnaires including the IC Problem Index (ICPI), IC symptom Index (ICSI), Pelvic Pain and Urgency/Frequency Patient Symptom Scale (PUF), and an Irritable Bowel Syndrome (IBS) questionnaire. This study specifically examined ICSI scores, because this score provides a representation of the burden of urologic symptoms over the previous thirty days. The ICSI has 4 questions, with answers on a scale from $0-5$, with 0 representing asymptomatic and 5 representing maximally symptomatic. The four questions address the following bladder symptoms over the previous month: urgency, frequency, nocturia, and pain/burning. Participants were also asked to complete surveys regarding prior medical and surgical history, demographics, and smoking status.

CWHD was performed in a standardized manner:

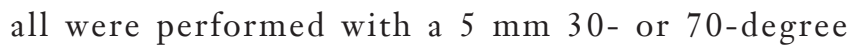
cystoscope, with sterile saline used as distending fluid. After $200 \mathrm{cc}$, the infusion was stopped and a survey of the bladder was performed. A minimum of two pictures were taken, one of the bladder bases and one of the domes of the bladder. Hydrodistension was then performed to gravity. The fluid bag was placed $80-100 \mathrm{~cm}$ above the bed level and bladder was filled to either capacity (documented) as determined by either leakage per the urethra or inability to further infuse fluid, or when $700 \mathrm{cc}$ was reached. Once the bladder was filled, it was then emptied within 60 seconds. At least five images were obtained: trigone, left bladder wall, right bladder wall, posterior bladder wall, and dome of the bladder.

Images were de-identified, batched in sets from 25 participants, and evaluated by a panel of 3 urogynecologists. Glomerulations were graded by distribution and number on a predetermined scale that was developed for this research project. The scale used in our analysis is as follows; $\geq 10$ glomerulations in 3-4 quadrants, $\geq 10$ glomerulations in 1-2 quadrants, glomerulations occurring at a lower rate than the previous 2 categories, or no glomerulations. The glomerulation category of a minimum of 10 glomerulations in 3 of 4 quadrants is based on the stringent National Institute of Diabetes and Digestive and Kidney Diseases (NIDDK) diagnosis for IC/BPS, which formed the basis for the research definition for IC/BPS (7). The next category, $\geq 10$ glomerulations in 1 or 2 quadrants, was selected at the discretion of the authors to represent a high rate of glomerulations that does not meet the NIDDK threshold.

As part of this analysis women who met the criteria for being minimally symptomatic or highly symptomatic were included in this study. Minimally symptomatic women were defined as women with a score of either 0 or 1 in all subscores of the ICSI, for a maximal score of 4 . Participants were also excluded from the minimally symptomatic cohort if they were originally scheduled for cystoscopy with hydrodistention rather than cystoscopy or if they had a history of IC/BPS; these patients were excluded because regardless of their current symptoms, original scheduling for CWHD signaled that a physician had diagnosed IC/ BPS. Highly symptomatic women were defined as women with a composite ICSI score of 12 or greater as well as with a score of 4 or 5 to the question: "Have you experienced pain or burning in your bladder?" We included the criteria of a pain/burning ICSI subscale score of 4 or greater, as we chose to focus on women with a high irritative symptom burden. No women with intermediate symptoms identified as part of the larger prospective study were included in this study. Study size was arrived at on the basis of above criteria. Bias was minimized by having all cystoscopic data de-identified and batched prior to analysis.

\section{Statistical analysis}

The primary outcome of this study was any glomerulations. Univariate associations were evaluated with Chi-square tests. Pairwise comparisons and subgroup analyses were carried out using Chi-square tests or Fisher's exact test. Secondary outcomes examined the demographics and medical history of our study groups. A power analysis was not performed prior to initiation of the study, as the cohorts examined in this study are part of a larger prospective study and all participants meeting criteria were included. A threshold $\mathrm{P}$ value of 0.05 was designated statistically significant. Incomplete data sets were excluded from analyses if they did not have ICSI scores or glomerulation scoring.

A sub-analysis was performed to evaluate women with either no glomerulations or $\geq 10$ glomerulations in 3 or 4 quadrants. Multivariable logistic regression was performed on the population in the subgroup analysis. Given the low event rate in the sub-analysis group, a series of logistic regressions were carried out in order to avoid over-fitting our model. The variables included in our model were minimal symptomatology, self-reported gastroesophageal 
reflux disease (GERD), recurrent urinary tract infection (rUTI), and history of endometriosis. These variables were selected as they noted to be statistically different between the minimally and maximally symptomatic group and were considered clinically-significant potential confounders. All analyses were performed using SPSS version 23.0 for Windows (IBM, Armonk, New York).

\section{Ethical statement}

The study was conducted in accordance with the Declaration of Helsinki (as revised in 2013). The study was approved by St. Louis University IRB board (number 255344) and informed consent was taken from all individual participants.

\section{Results}

The overarching prospective data set included 667 participants. From that data set, we selected for minimally and maximally symptomatic women. We excluded the following participants from the analysis: participants in whom a glomerulations category was unknown (no reviewer data) or incomplete (with one reviewer's data only) or in which there was not concordance among at least two of three image reviewers. We excluded participants in whom O'Leary/Sant questionnaire scores were unknown. We excluded participants who withdrew from the study given that withdrawal resulted in lack of cystoscopic data. Of the 667 women who had cystoscopy and hydrodistension, 84 met the inclusion criteria for this study. Thirty-three comprised the "minimally symptomatic" cohort and 51 met criteria for the "highly symptomatic" cohort (Figure 1).

Patients in the two groups had similar age and BMI (Table 1). More women in the highly symptomatic group were Caucasian $(\mathrm{P}<0.05)$. Women in the minimally symptomatic group were significantly less likely to have the following self-reported conditions: GERD, IBS, rUTI, fibromyalgia, and endometriosis. The two groups were not significantly different in regards to having diabetes, thyroid disorder, autoimmune disorders, chronic hypertension, menopausal status, gynecologic surgical history, or parity. No complications were reported as associated with cystoscopy with hydrodistension. Overall complication rate and type was not collected as part of this study.

When assessing for the primary outcome of "any glomerulations", the study population was divided into two glomerulation categories: no glomerulations vs. any level of glomerulations. There was no difference in any glomerulations seen between minimally symptomatic women and highly symptomatic women $[9 / 33$ (27.3\%) vs. $21 / 51(41.2 \%), \mathrm{P}=0.29]$.

When comparing the distribution of glomerulations across our two cohorts, minimally symptomatic women were less likely to have significant glomerulations $(\geq 10$ glomerulations in 3 or 4 quadrants) than women who had high symptomatology, ( $3.0 \%$ vs. $23.5 \%, \mathrm{P}<0.05$, Table 2$)$.

We performed a subgroup analysis to evaluate women with the two extremes of glomerulations: women with either $\geq 10$ glomerulations in 3 or 4 quadrants or no glomerulations. There were 67 women in the subgroup analysis; 25 minimally symptomatic and 42 highly symptomatic. Patients in the two sub-analysis groups also similar age, race, and BMI (Table 3). Women in the minimally symptomatic group were significantly less likely to have the following self-reported conditions: GERD, IBS, rUTI, and endometriosis. Minimally symptomatic women were significantly less likely to have $\geq 10$ glomerulations in 3 or 4 quadrants compared to highly symptomatic women in a pairwise comparison of the subgroup analysis $[1 / 25(4.0 \%)$ vs. $12 / 42(28.6 \%), \mathrm{P}<0.05]$.

We performed multivariable logistic regression analyses on the sub-analysis group of 67 women with no glomerulations or $\geq 10$ glomerulations in 3 or 4 quadrants to account for baseline differences between the two groups (Tables 4-6). Due to low event rates, we created 3 models, as the number of covariates we could include in each model was limited. Each regression model assessed the association between minimal symptomatology and $\geq 10$ glomerulations in 3 or 4 quadrants when controlling for one of the following variables: GERD, endometriosis, and rUTI. In the first model, minimally symptomatic women were less likely to have $\geq 10$ glomerulations in 3 or 4 quadrants when controlling for GERD [adj. OR 0.1077 (0.012-0.928); $\mathrm{P}<0.05]$. Similarly, in the second model, when controlling for endometriosis, minimally symptomatic women were less likely to have $\geq 10$ glomerulations in 3 or 4 quadrants [adj. OR $0.069(0.008-0.589) ; \mathrm{P}<0.05]$. However, in the third model when controlling for recurrent UTI, the association between minimally symptomatic women and $\geq 10$ glomerulations in 3 or 4 quadrants was no longer significant [adj. OR 0.132 (0.015-1.134); P=0.07]. Neither GERD, endometriosis, nor rUTI had significant associations with $\geq 10$ glomerulations in 3 or 4 quadrants in any of the models. 


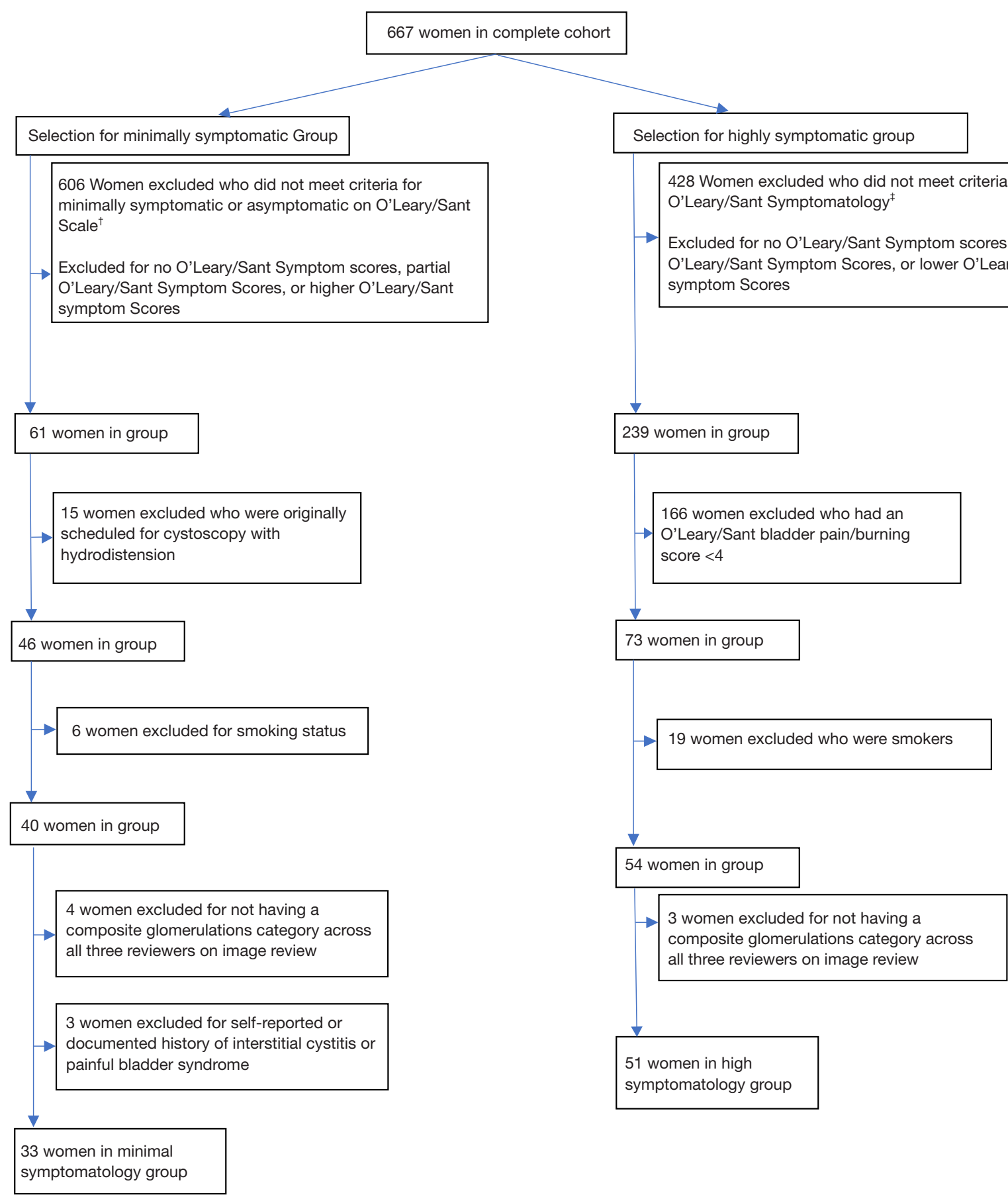

Figure 1 Selection of study groups. ${ }^{\dagger}, 606$ women were excluded in the following manner: 83 for no ICSI scores, 6 for partial O’Leary-Sant Symptom Score (ICSI) scores, and 517 for higher ICSI scores (ICSI scores $\geq 12$ ). ${ }^{\ddagger}, 428$ women were excluded in the following manner: 83 for no ICSI score, 6 for partial ICSI scores, and 339 for lower ICSI scores (ICSI score $<12$ ). 
Table 1 Demographics and medical history of participants

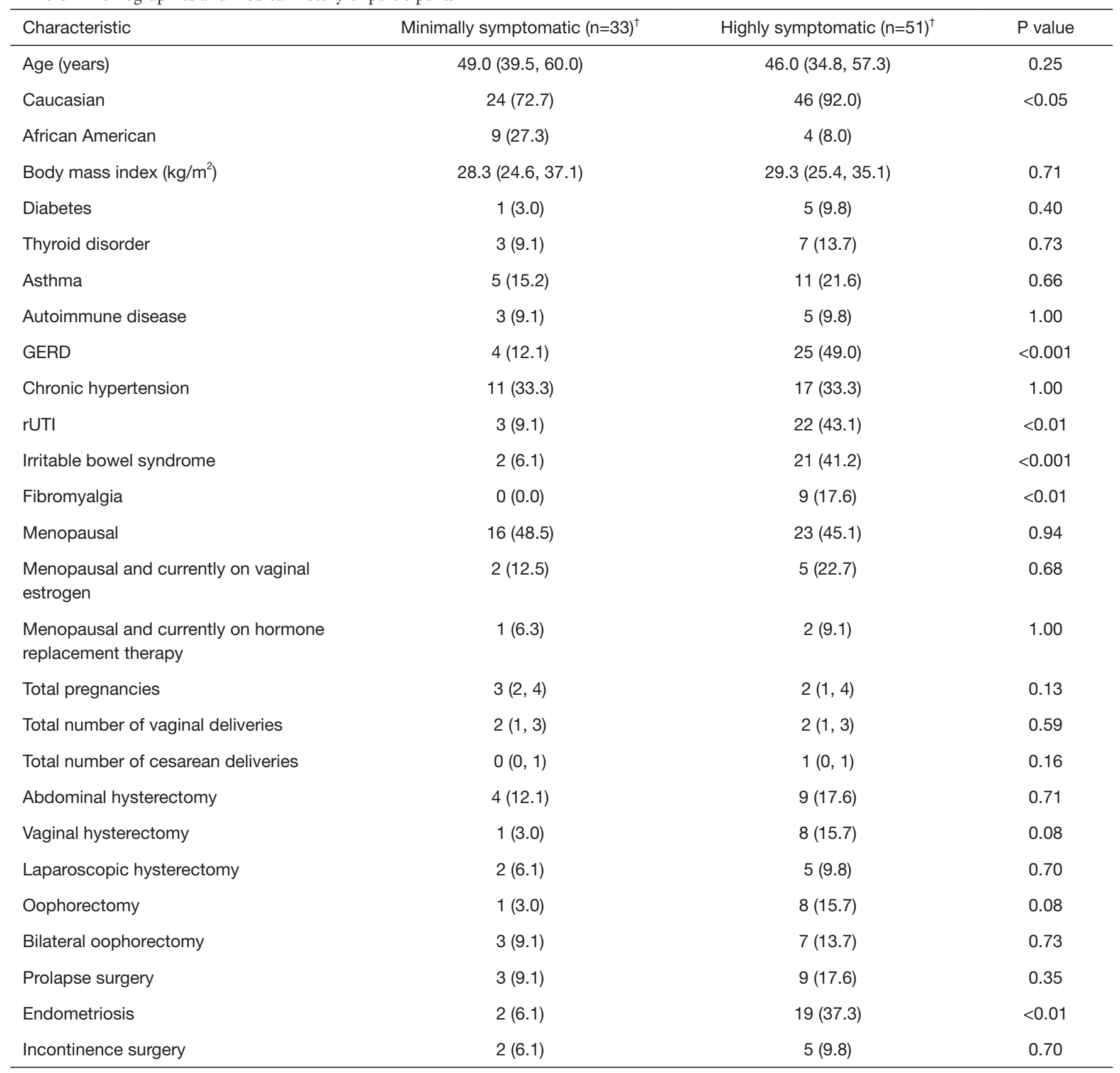

${ }^{\dagger}$, data are reported as medians, interquartile ranges (IQR) or number, percentage.

Table 2 Glomerulation distribution for women with minimal vs. high symptomatology

\begin{tabular}{lccrr}
\hline Symptom category & $\begin{array}{c}\geq 10 \text { glomerulations in } 3 \\
\text { or } 4 \text { quadrants, } n(\%)^{\dagger}\end{array}$ & $\begin{array}{c}\geq 10 \text { glomerulations in } 1 \\
\text { or } 2 \text { quadrants, } n(\%)^{\dagger} \text { occurring at a lower rate, } \mathrm{n}(\%)^{\dagger}\end{array}$ & $\begin{array}{c}\text { Any glomerulations } \\
\mathrm{n}(\%)^{\dagger}\end{array}$ & \multicolumn{2}{c}{$\begin{array}{c}\text { No glomerulations, } \mathrm{P} \text { value } \\
\text { Minimally symptomatic }(\mathrm{n}=33)\end{array}$} & $1(3.0 \%)$ & $5(15.2 \%)$ & $24(72.7 \%)$ & $<0.05$ \\
Highly symptomatic $(\mathrm{n}=51)$ & $12(23.5 \%)$ & $6(11.8 \%)$ & $3(5.9 \%)$ & $30(58.8 \%)$ \\
\hline
\end{tabular}

\footnotetext{
${ }^{\dagger}$, data are reported as number, percentage.
} 
Table 3 Demographics and medical history of participants in subgroup analysis

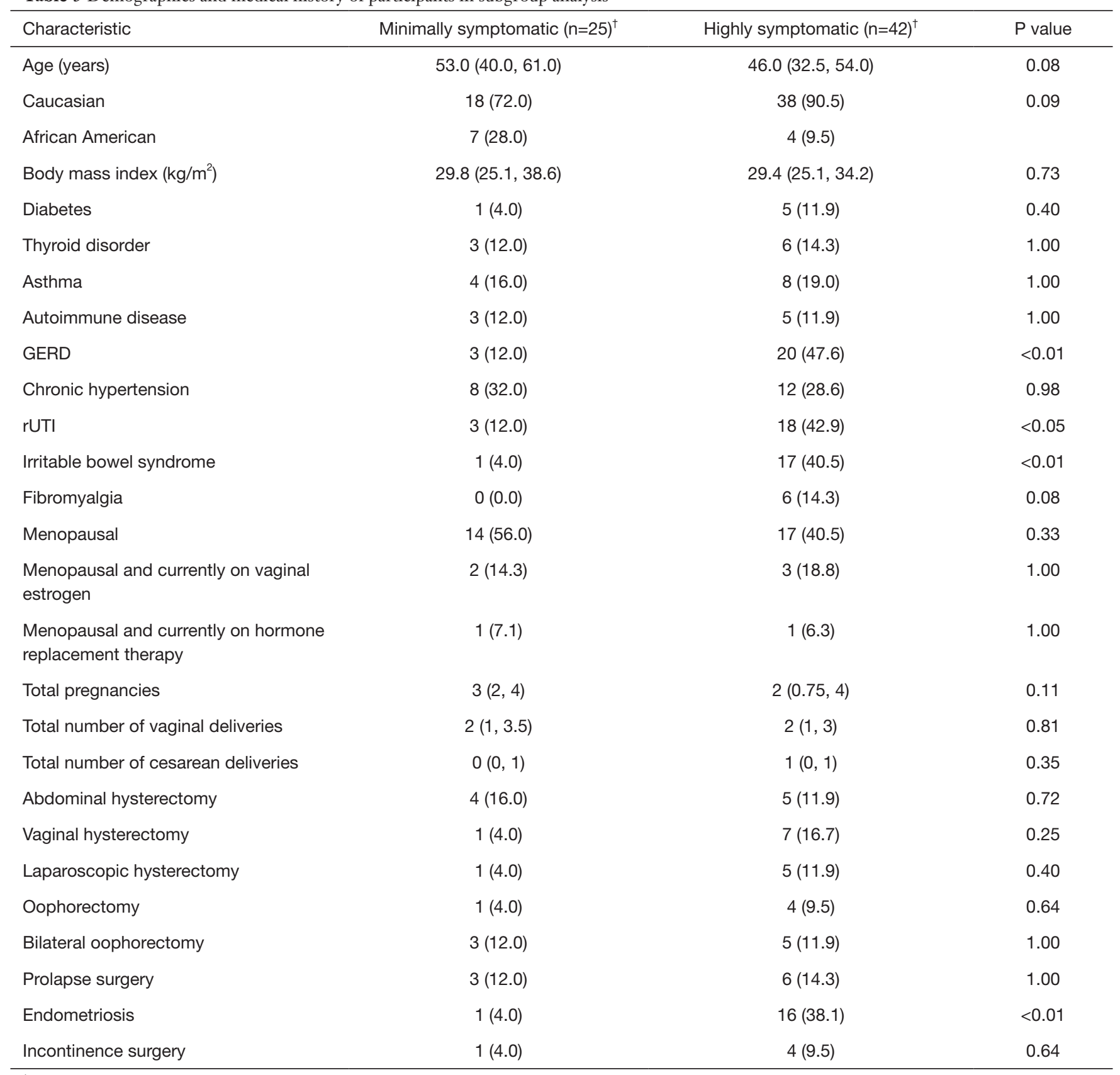

${ }^{\dagger}$, data are reported as medians, interquartile ranges (IQR) or number, percentage.

Table 4 Logistic regression models for $\geq 10$ glomerulations in 3 or 4 quadrants in sub-analysis population: bivariate logistic regression model of minimal symptomatology and GERD for $\geq 10$ glomerulations in 3 or 4 quadrants in sub-analysis population ${ }^{\dagger}$

\begin{tabular}{lccc}
\hline Variable & Adjusted odds ratio & $95 \% \mathrm{Cl}$ & $\mathrm{P}$ value \\
\hline Minimal symptomatology & 0.107 & $0.012-0.928$ & $<0.05$ \\
GERD & 1.077 & $0.291-3.981$ & 0.91 \\
\hline
\end{tabular}

\footnotetext{
${ }^{\dagger}$, sub-analysis population are the 67 women with no glomerulations and those with $\geq 10$ glomerulation in 3 or 4 quadrants.
} 
Table 5 Logistic regression models for $\geq 10$ glomerulations in 3 or 4 quadrants in sub-analysis population: bivariate logistic regression model of minimal symptomatology and endometriosis for $\geq 10$ glomerulations in 3 or 4 quadrants in sub-analysis population ${ }^{\dagger}$

\begin{tabular}{lccc}
\hline Variable & Adjusted odds ratio & $95 \% \mathrm{Cl}$ & $\mathrm{P}$ value \\
\hline Minimal symptomatology & 0.069 & $0.008-0.589$ & $<0.05$ \\
Endometriosis & 0.227 & $0.042-1.213$ & 0.08 \\
\hline
\end{tabular}

${ }^{\dagger}$, sub-analysis population are the 67 women with no glomerulations and those with $\geq 10$ glomerulation in 3 or 4 quadrants.

Table 6 Logistic regression models for $\geq 10$ glomerulations in 3 or 4 quadrants in sub-analysis population: bivariate logistic regression model of minimal symptomatology and recurrent UTI for $\geq 10$ glomerulations in 3 or 4 quadrants in sub-analysis population ${ }^{\dagger}$

\begin{tabular}{lccc}
\hline Variable & Adjusted odds ratio & $95 \% \mathrm{Cl}$ & $\mathrm{P}$ value \\
\hline Minimal symptomatology & 0.132 & $0.015-1.134$ & 0.07 \\
rUTI & 2.172 & $0.586-8.052$ & 0.25 \\
\hline
\end{tabular}

${ }^{\dagger}$, sub-analysis population are the 67 women with no glomerulations and those with $\geq 10$ glomerulation in 3 or 4 quadrants.

\section{Discussion}

Our study shows a significant difference in the distribution of glomerulations in minimally and highly symptomatic women. While there was not a difference in our primary outcome of 'any glomerulations' between minimally and highly symptomatic women, comparison of the distribution of glomerulation between minimally and highly symptomatic women as well as the pairwise comparison examining only women at the extremes of visualized glomerulations (with no glomerulations or $\geq 10$ glomerulations in 3 or 4 quadrants) did show minimally symptomatic women were significantly less likely to have $\geq 10$ glomerulations in 3 or 4 quadrants compared to highly symptomatic women. This reflects that the significant difference in the glomerulation distribution between our two groups lies at the extremes of glomerulation categories. The significant association between minimal symptomatology and no glomerulations in the univariate subgroup analysis persisted in the multivariable logistic regression analysis when controlling for GERD and endometriosis, but not when controlling for rUTI.

This study contributes to the discussion surrounding the clinical role of glomerulations. Glomerulations have been reported in patients with alternative diagnoses to IC/ BPS $(5,6,16)$. However, there is limited prospective data examining asymptomatic or minimally symptomatic women. Glomerulations on CWHD were found in asymptomatic, normal women undergoing tubal ligation at the same rate as pictures of historical controls with a diagnosis of IC/BPS in a study by Waxman and colleagues (12). Our study's findings contrast with those above as we found that minimally symptomatic women are significantly less likely to have a high glomerulation burden compared to highly symptomatic women.

We used potential risk factors for glomerulations in creating our logistic regression models. Recurrent urinary tract infections were included in the model, as they can be a frequent misdiagnosis, but also may contribute to the development of IC/BPS and urothelial damage (19). Endometriosis was selected as it is a common comorbidity of IC/BPS that results in lesions and upregulation of neuroinflammatory processes (20-22). We included GERD in the model because of an association of functional dyspepsia with IC/BPS, combined with similarities in urothelium and esophageal mucosa in those with glomerulations and erosions respectively $(23,24)$.

The recognition of overarching chronic pain syndromes casts new light on the debate regarding cystoscopic findings, especially in regards to patients with glomerulations with other diagnoses (25). A recent systematic review of cystoscopic findings as it relates to IC/BPS concluded that glomerulations are not specific for IC/BPS and can be found in patients with LUTS, benign prostatic hypertrophy, overactive bladder (OAB), prostatitis, and urolithiasis $(3,4,6,9,11,26,27)$. Glomerulations also may be a consequence of multiple bladder pathologies. This understanding may be useful in patients with chronic pelvic pain with multiple etiologies, such as endometriosis, myofascial pain, and others. While we would not advocate for routine cystoscopy with hydrodistention in assessment of LUTS or for the use of glomerulations as diagnostic 
criteria, there may be a role for bladder assessment in patients with complex and overlapping pelvic symptoms and in patients in whom the contribution of bladder sensitivity to overall symptoms is uncertain. If glomerulations are associated with bladder pathology, irrespective of a specific diagnosis, cystoscopy with hydrodistention might prompt clinicians towards more aggressive treatment of potential sources of bladder pain. This comes with the caveat that lack of glomerulations does not mean bladder health, just as lack of any one specific pathologic sign or symptom does not signify bladder health.

A strength of this study was that a specific definition of IC/BPS was not employed, due to the breadth of society definitions in diagnosis. Instead this study focused on symptom burden $(13,28)$. We acknowledge that this serves as an imperfect, but generalizable, surrogate for a spectrum of low symptomatology we would consider "normal". Conversely, we defined our "highly symptomatic group" as having an ICSI score cutoff of 12 or greater, as this has been previously used as a great burden of IC/PBS-associated symptoms $(29,30)$. The use of the ICSI as part of assessment of broader LUTS, not just IC/BPS, is supported by its use in the Multidisciplinary Approach to the Study of Chronic Pelvic Pain (MAPP) study (25).

A further strength of our study is that the reviewers of the images were blinded to preoperative diagnosis, evaluated the images separately, and there had to be consensus amongst them on the images.

The limitations of this study include ranking glomerulations along an unvalidated, predetermined scale. However, we note that the category with most glomerulations is NIDDK criteria for IC/BPS based on cystoscopic findings.

Therefore, a further direction of study lies in assessing whether there is a certain burden of glomerulations that is associated with disease states. The NIDDK research criteria may not fit with a developing understanding of the clinical relevance of glomerulations (7). Further investigations are needed to assess the relationship between glomerulations and broader bladder pathology. In the setting in which glomerulations are not used for diagnosis of a certain disease condition, but rather as a marker of an unhealthy bladder, they would prompt a clinician to investigate for clinical bladder symptoms.

\section{Conclusions}

While glomerulations are no longer used in the diagnosis of IC/BPS, minimally symptomatic women only rarely have a significant glomerulation burden $(3,11,29)$. There is little data to support that glomerulations are present in asymptomatic patients (10). That is to say, that although glomerulations may not be limited to IC/BPS, they may represent a pathologic state. Incidentally found glomerulations ought to prompt investigation regarding a patient's urinary symptoms as there is a significant possibility of revealing lower urinary tract pathology. The understanding of whether or not glomerulations represent a pathological bladder state is important to the clinician, as we seek to define both disease bladder states and the definitions of bladder health.

\section{Acknowledgments}

Thanks for Collin Miller and Jim Rice for contributions to database building and management.

Funding: None.

\section{Footnote}

Reporting Checklist: The authors have completed the STROBE reporting checklist. Available at https://dx.doi. org/10.21037/tau-21-195

Data Sharing Statement: available at https://dx.doi. org/10.21037/tau-21-195

Peer Review File: Available at https://dx.doi.org/10.21037/ tau-21-195

Conflicts of Interest: All authors have completed the ICMJE uniform disclosure form (available at https://dx.doi. org/10.21037/tau-21-195). The authors have no conflicts of interest to declare.

Ethical Statement: The authors are accountable for all aspects of the work in ensuring that questions related to the accuracy or integrity of any part of the work are appropriately investigated and resolved. The study was conducted in accordance with the Declaration of Helsinki (as revised in 2013). The study was approved by St. Louis University IRB board (number 255344) and informed consent was taken from all individual participants.

Open Access Statement: This is an Open Access article distributed in accordance with the Creative Commons 
Attribution-NonCommercial-NoDerivs 4.0 International License (CC BY-NC-ND 4.0), which permits the noncommercial replication and distribution of the article with the strict proviso that no changes or edits are made and the original work is properly cited (including links to both the formal publication through the relevant DOI and the license). See: https://creativecommons.org/licenses/by-nc-nd/4.0/.

\section{References}

1. McLennan MT. Interstitial Cystitis. Obstet Gynecol Clin North Am 2014:41:385-95.

2. Davis NF, Brady CM, Creagh T. Interstitial cystitis/painful bladder syndrome: epidemiology, pathophysiology and evidence-based treatment options. Eur J Obstet Gynecol Reprod Biol 2014;175:30-7.

3. Wennevik GE, Meijlink JM, Hanno P, et al. The Role of Glomerulations in Bladder Pain Syndrome: A Review. J Urol 2016;195:19-25.

4. Berger RE, Miller JE, Rothman I, et al. Bladder petechiae after cystoscopy and hydrodistension in men diagnosed with prostate pain. J Urol 1998;159:83-5.

5. Awad SA, MacDiarmid S, Gajewski JB, et al. Idiopathic reduced bladder storage vs. interstitial cystitis. J Urol 1992;148:1409-12.

6. Hassan AA, Elgamal SA, Sabaa MA, et al. Evaluation of intravesical potassium sensitivity test and bladder biopsy in patients with chronic prostatitis/chronic pelvic pain syndrome. Int J Urol 2007;14:738-42.

7. Gillenwater JY, Wein AJ. Summary of the National Institute of Arthritis, Diabetes, Digestive and Kidney Diseases Workshop on Interstitial Cystitis, National Institutes of Health, Bethesda, Maryland, August 28-29, 1987. J Urol 1988;140:203-6.

8. Hanno PM, Landis JR, Matthews-Cook Y, et al. The diagnosis of interstitial cystitis revisited: lessons learned from the National Institutes of Health Interstitial Cystitis Database study. J Urol 1999;161:553-7.

9. Furuya R, Masumori N, Furuya S, et al. Glomerulation Observed During Transurethral Resection of the Prostate for Patients With Lower Urinary Tract Symptoms Suggestive of Benign Prostatic Hyperplasia is a Common Finding But No Predictor of Clinical Outcome. Urology 2007;70:922-6.

10. Chung MK. Interstitial cystitis in persistent posthysterectomy chronic pelvic pain. JSLS 2004;8:329-33.

11. Jiang YH, Kuo HC. Poster Session: PO-07: Development of Bladder Glomerulations after Hydrodistension in Patients with Upper Urinary Tract Urolithiasis Suggesting Cross Talk and Bladder Inflammation Occur between Upper and Lower Urinary Tract. Int J Urol 2014;21:A12-24.

12. Waxman JA, Sulak PJ, Kuehl TJ. Cystoscopic findings consistent with interstitial cystitis in normal women undergoing tubal ligation. J Urol 1998;160:1663-7.

13. Hanno PM, Burks DA, Clemens JQ, et al. AUA guideline for the diagnosis and treatment of interstitial cystitis/ bladder pain syndrome. J Urol 2011;185:2162-70.

14. Fall M, Baranowski AP, Elneil S, et al. EAU Guidelines on Chronic Pelvic Pain. Eur Urol 2010;57:35-48.

15. Abrams P, Cardozo L, Fall M, et al. The standardisation of terminology in lower urinary tract function: report from the standardisation sub-committee of the International Continence Society. Urology 2003;61:37-49.

16. Temml C, Wehrberger C, Riedl C, et al. Prevalence and correlates for interstitial cystitis symptoms in women participating in a health screening project. Eur Urol 2007;51:803-8; discussion 809.

17. Kennedy CM, Bradley CS, Galask RP, et al. Risk factors for painful bladder syndrome in women seeking gynecologic care. Int Urogynecol J Pelvic Floor Dysfunct 2006; 17:73-8.

18. Song $\mathrm{Y}$, Zhang $\mathrm{W}, \mathrm{Xu} \mathrm{B}$, et al. Prevalence and correlates of painful bladder syndrome symptoms in Fuzhou Chinese women. Neurourol Urodyn 2009;28:22-5.

19. Warren JW, Howard FM, Cross RK, et al. Antecedent nonbladder syndromes in case-control study of interstitial cystitis/painful bladder syndrome. Urology 2009;73:52-7.

20. Bogart LM, Berry SH, Clemens JQ. Symptoms of interstitial cystitis, painful bladder syndrome and similar diseases in women: a systematic review. J Urol 2007;177:450-6.

21. Stanford EJ, Koziol J, Feng A. The prevalence of interstitial cystitis, endometriosis, adhesions, and vulvar pain in women with chronic pelvic pain. J Minim Invasive Gynecol 2005;12:43-9.

22. Alagiri M, Chottiner S, Ratner V, et al. Interstitial cystitis: Unexplained associations with other chronic disease and pain syndromes. Urology 1997;49:52-7.

23. Chelimsky G, Heller E, Buffington CAT, et al. CoMorbidities of Interstitial Cystitis. Front Neurosci 2012;6:114.

24. Birder LA, Hanna-Mitchell AT, Mayer E, et al. Cystitis, Co-morbid disorders and associated epithelial dysfunction. Neurourol Urodyn 2011;30:668-72.

25. Landis JR, Williams DA, Lucia MS, et al. The MAPP 
research network: design, patient characterization and operations. BMC Urol 2014;14:58.

26. Huang J, Zheng Z, Wei H, et al. A comparative study of the laparoscopic appearance and anatomy of the autonomic nervous in normal males. Zhonghua Wai Ke Za Zhi 2014;52:500-3.

27. Chung MK, Butrick CW, Chung CW. The Overlap of Interstitial Cystitis/Painful Bladder Syndrome and Overactive Bladder. JSLS 2010;14:83-90.

28. van de Merwe JP, Nordling J, Bouchelouche P, et al. Diagnostic criteria, classification, and nomenclature for

Cite this article as: Marcu I, Gavard JA, Vazirabadi G, Shi JY, Steele AC, Leong FC, McLennan MT, Bickhaus JA, Holloran-Schwartz MB, Yeung P Jr, Campian EC. Cystoscopic findings in women with minimal and maximal lower urinary tract symptoms. Transl Androl Urol 2021;10(7):2910-2920. doi: 10.21037/tau-21-195 painful bladder syndrome/interstitial cystitis: an ESSIC proposal. Eur Urol 2008;53:60-7.

29. Berry SH, Bogart LM, Pham C, et al. Development, validation and testing of an epidemiological case definition of interstitial cystitis/painful bladder syndrome. J Urol 2010;183:1848-52.

30. Wyndaele JJ, Van Dyck J, Toussaint N. Cystoscopy and bladder biopsies in patients with bladder pain syndrome carried out following ESSIC guidelines. Scand J Urol Nephrol 2009;43:471-5. 